\title{
THE OUTLOOK AND CHALLENGES OF “IT" APPLICATIONS FOR GAS PIPELINES MAINTENANCE OPERATIONS
}

\author{
Kohei Kawano \\ Development \& Sales Department \\ Tokyo Gas Engineering co., ltd. \\ Kamata5-37-1,Ota-ku,Tokyo \\ kawano@tge.co.jp
}

\begin{abstract}
Tokyo Gas Co., ltd, the largest gas company in Japan, supplies city gas to Tokyo Metropolitan area, covering $3000 \mathrm{~km} 2$ and 9.6million customers. Increasing maintenance cost and damages of underground utilities let Tokyo Gas start the research and development of digital mapping in 1977. Tokyo Gas developed GIS basic software "TUMSY" in 1983 after the several years' research of foreign GIS products, which could not afford to store huge and dense pipeline-drawings. GIS basic software TUMSY consisted of DBMS and business applications. By using TUMSY, 30,000 manual drawings were digitized into the database. Then for about 20 years, TUMSY has been reinforced and improved on the same architecture, and various applications with utilization of database have been developed. The history tells us that GIS is essential to the company activities, and must be always implemented on the highest level of technology and business expertise.
\end{abstract}

Keywords: GIS, Mobile computing, CALS/EC, GPS

\section{TOKYO GAS PROFILE}

Tokyo Gas is the largest gas company in Japan, and located in the southern area of Kanto region including Metropolitan Tokyo, covering $3000 \mathrm{~m} 2$ for the supply area. It was founded in 1885 , and has been the largest gas company in Japan.

(Fig.1)

. The number of customers is 9.6 millions, Gas sales volume is 11,934 million $\mathrm{m} 3 / 46.04655 \mathrm{MJ}$, and the length of Mains is $50,808 \mathrm{~km}$. ( March 31, 2005)

The business scope is from Gas production (from LNG), Transmission, Distribution, to Gas \& gas appliances sales and Customer service.

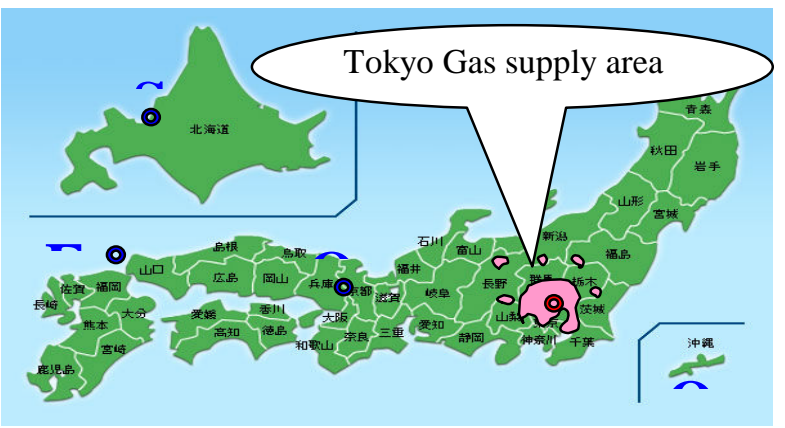

Fig1. Tokyo Gas supply area

\section{DEVELOPMENT OF TUMSY}

\subsection{Background of the development}

a) Before 1980s, almost all jobs were manual-based from pipeline designs to maintenance operations in Tokyo Gas. It took much effort to improve efficiency and safety in the pipeline day-to-day work. (Fig.2)

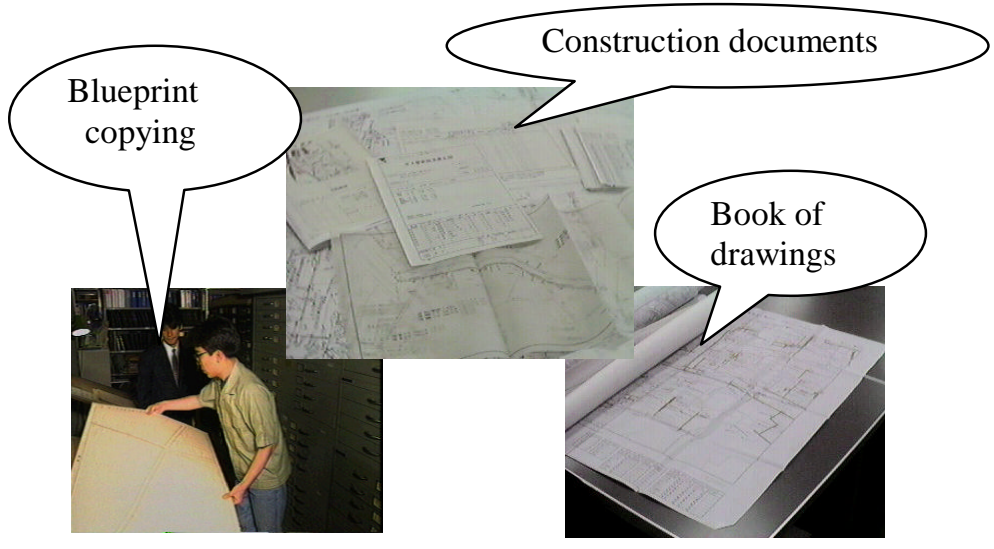

Fig2. manual drawings

b) Especially in Tokyo metropolitan area, congested roads both above and under the ground made it difficult to maintain manual pipeline drawings.(Fig.3)

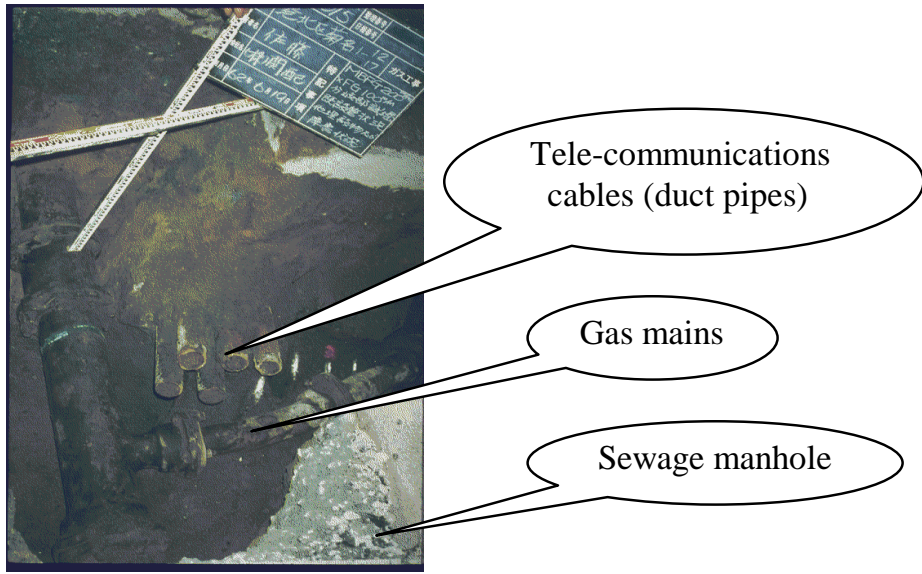

Fig3. A congested road under the ground in central Tokyo 
c)In Japan, the government strongly directed utilities to keep accurate facilities drawings for safety. Increasing maintenance cost and damages of underground utilities let Tokyo Gas start the research and development of digital mapping.

\subsection{Steps of GIS development}

Steps of GIS development fall into four periods as follows: 1977-1990

-R\&D of GIS basic software TUMSY,

-Conversion of 30,000 drawings

1991-1998

-Advanced utilization of database

1999-2004

-Introduction of IT new technology 2005-:

-Total system renewal (Now implementing)

a) 1977-1990: R\&D and Conversion

GIS basic software TUMSY was developed in 1983 by Tokyo Gas after the several years' research of foreign GIS products from IBM or CALMA which could not afford to store dense Tokyo Gas pipeline drawings.

TUMSY specifications in 1983 are as follows:

-Host computer: VAX-11/780

-Operating system: VMS

-Database: VMS/RMS(record management system)

-Memory: $1 \mathrm{MB}$,

-Disk: 300MB

-Graphic display: JRC (Japan Radio Co, ltd.)etc

GIS basic software TUMSY consisted of:

-Database management software,

-Mapping handling software,

-Graphic processing software

TUMSY basic applications are:

-Retrieval system,

-Drawing Plotting system,

-Network analysis,

-Data input/update (Fig.4)

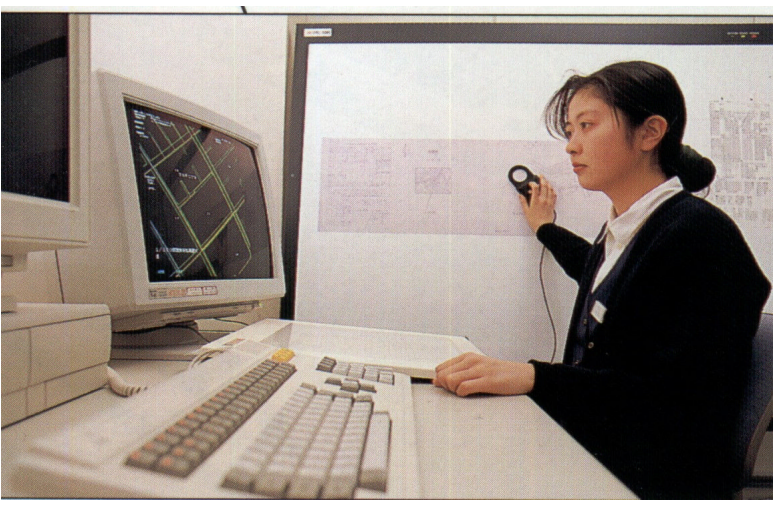

Fig.4 Data input/update operation
By using TUMSY, 30,000 manual drawings were digitized into the database from 1984 to 1986.(Fig,5)

Manual drawings were converted to layered database consisting of Gas-mains, Services, Valves, Regulators, Meters, Buildings, Householder name, Roads etc.

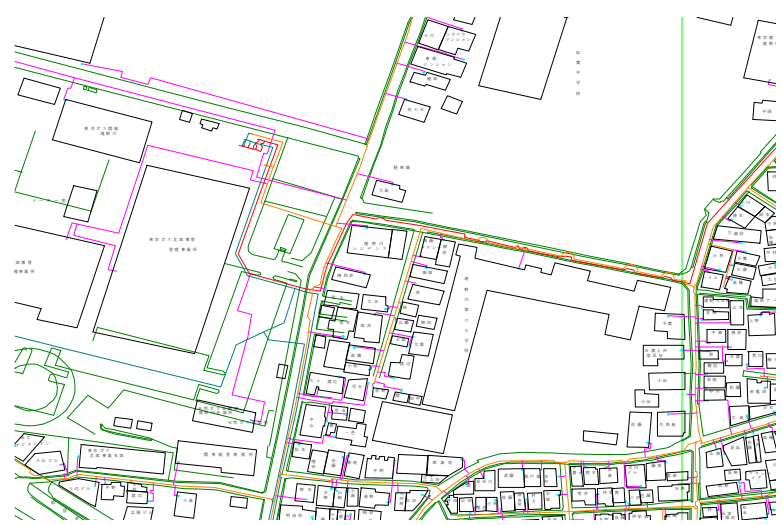

Fig.5 1/500 digitized drawing

In 1988, GIS database was connected to Customer DB via SNA Gateway, and customer services were improved for quick response to customers' inquiries by mapping retrieval. From 1988, ROADIS (nationwide GIS project for roads and utilities) has started, and TUMSY was connected to ROADIS for data retrieval and exchange.

b) 1991-1998: Advanced utilization of database

In this period, $\mathrm{PC}$ technology was greatly advanced by "WINTEL", as keywords, "Windows, Mobile computing, multi-media data processing".

TUMSY was upgraded to those specifications, and various applications were continuously developed.

-gas emergency dispatch support system (Fig.6)

-gas supply operation support system (Fig.7)

-gas appliance service support system

-business strategy mapping system 


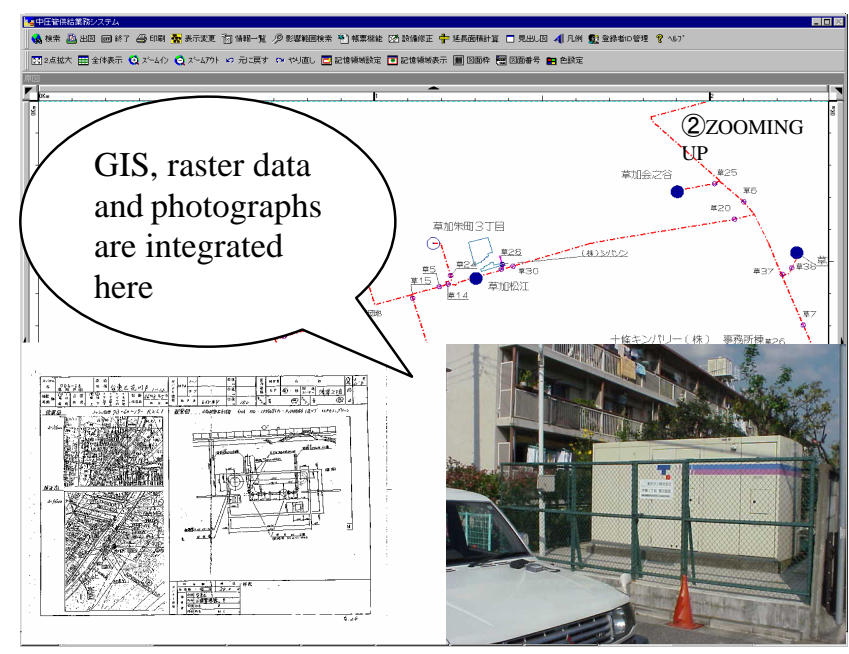

Fig.7 gas supply operation support system (GSOSS)

GSOSS improves the quality and efficiency of maintenance work flow, by cutting time and space for documents arrangement and manual planning.

c) 1999-2004: Introduction of IT new technology

In this period, "IT" technology was totally innovated by broadband networking, Internet Technology, mobile phone systems, and cost-down of IT TCO (Total Cost of Ownership).

TUMSY was enhanced to meet new technology specifications, and various applications were timely developed.

-Web GIS “TUMSY-Supera” development (Fig.8)

-Mobile GIS development (Fig.9)

-CALS/ EC data cycle application (Fig.10)

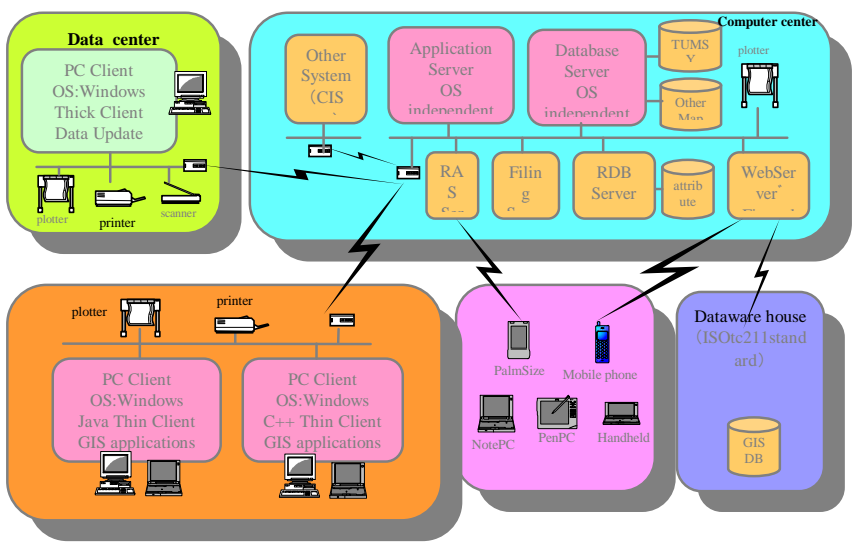

Fig.8 Web GIS “TUMSY-Supera” system configuration

Features of TUMSY-Supera are as follows:

-designed on concept of OOD (Object Oriented Design).

-3 layers architecture (DB sever, application sever, client) -data is communicated by http protocol which covers from desktop PCs to mobile phones.

-succeeding to TUMSY software heritage.

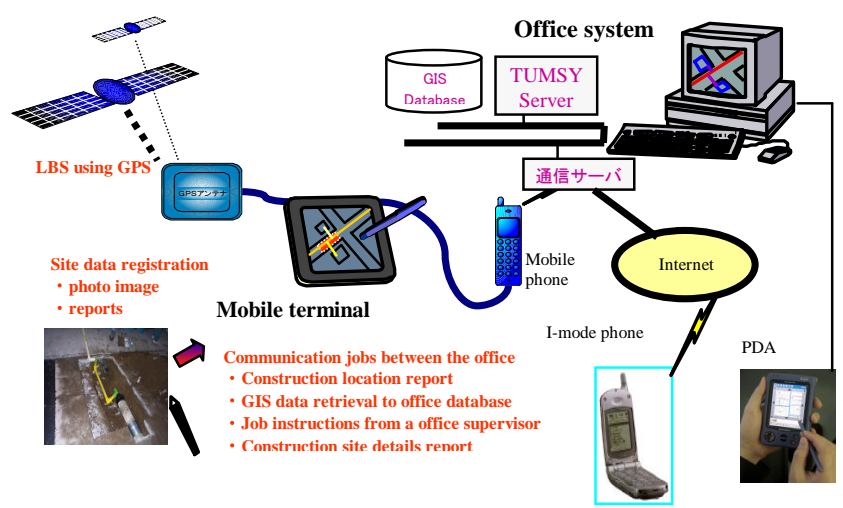

Fig.9 Mobile GIS development

Features of Mobile GIS are as follows:

-integrated through offices to fields.

-various mobile devices (pen PC, PDA, mobile phone) -succeeding to TUMSY software heritage.

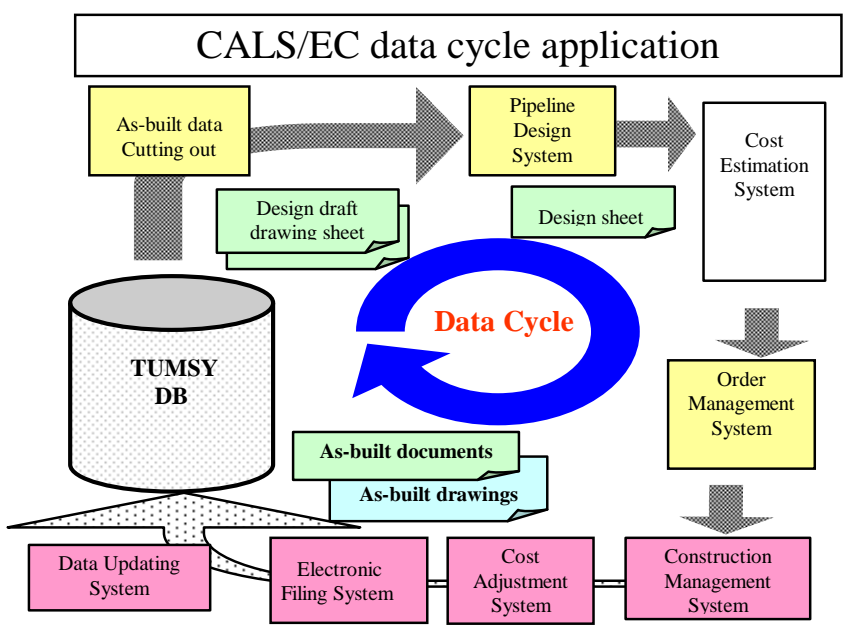

Fig.10 CALS/ EC data cycle application

Features of CALS/EC data cycle application are as follows: -drawing data exchange format is DXF or SXF/XML -The system is networked between Tokyo Gas and contractors.

The System configuration of Tokyo Gas GIS is: -GIS server: 2Alpha servers, 10unix servers -Clients: 400 Thick clients, 5000 thin clients, 1000mobile clients

-24hours x 365 days service is available

Additionally, TUMSY has also been introduced into more than 100 users in Japan (Gas users 34, Water 44, Sewage 7, ROADIS 7 and so on) through Tokyo Gas engineering co., ltd.. 
d) 2004-: Total system renewal

Total system renewal has been planned, and stated in 2005 . HP alpha processors will fade out into Intel Itanium. TUMSY architecture are still effective for utility GIS, but utilization of innovative GIT (Geospatial Information Technology) needs a total system renewal of TUMSY including the migration to Itanium. The renewal will end at 2007.

Features of total system renewal are as follows: -Operating systems are HP-UX and Windows -Introduction of Spatial DB (based on OGC standard) -Open Source approach ( MySQL etc.)

-SOA (Service Oriented Architecture) approach "GeOAP" which covers EUC/GIS users.(Fig.11)

-Web technology updating (Ajax etc.)

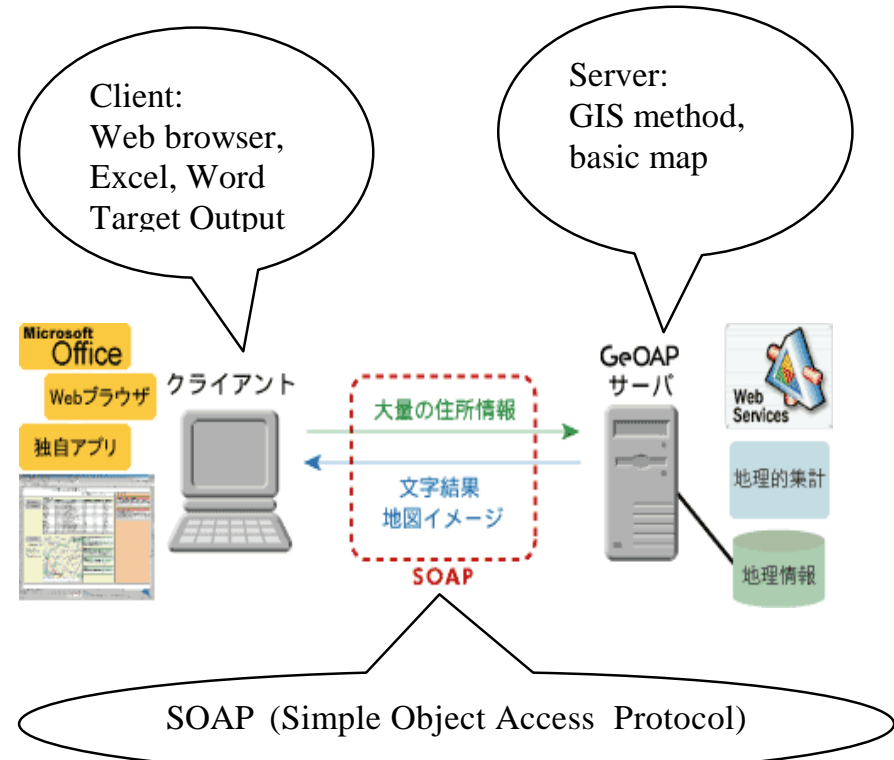

Fig.11 Web services "GeOAP"

e)Near future - Robot technology \& GIS Integration

In the field of Gas pipeline engineering, various robot technologies have been developed and utilized as follows: -pipeline internal inspection system -pipeline internal repair system -pipeline trenchless installation system

These technologies need the geometry and location of target pipelines, and GIS \& CAD can provide it accurately. At the same time, the result of inspections or operations can be obtained automatically as electronic drawings and documents.

Fig.12 shows a fiberscope system for gas pipelines. GIS will be integrated with the device and utilized in the near future.

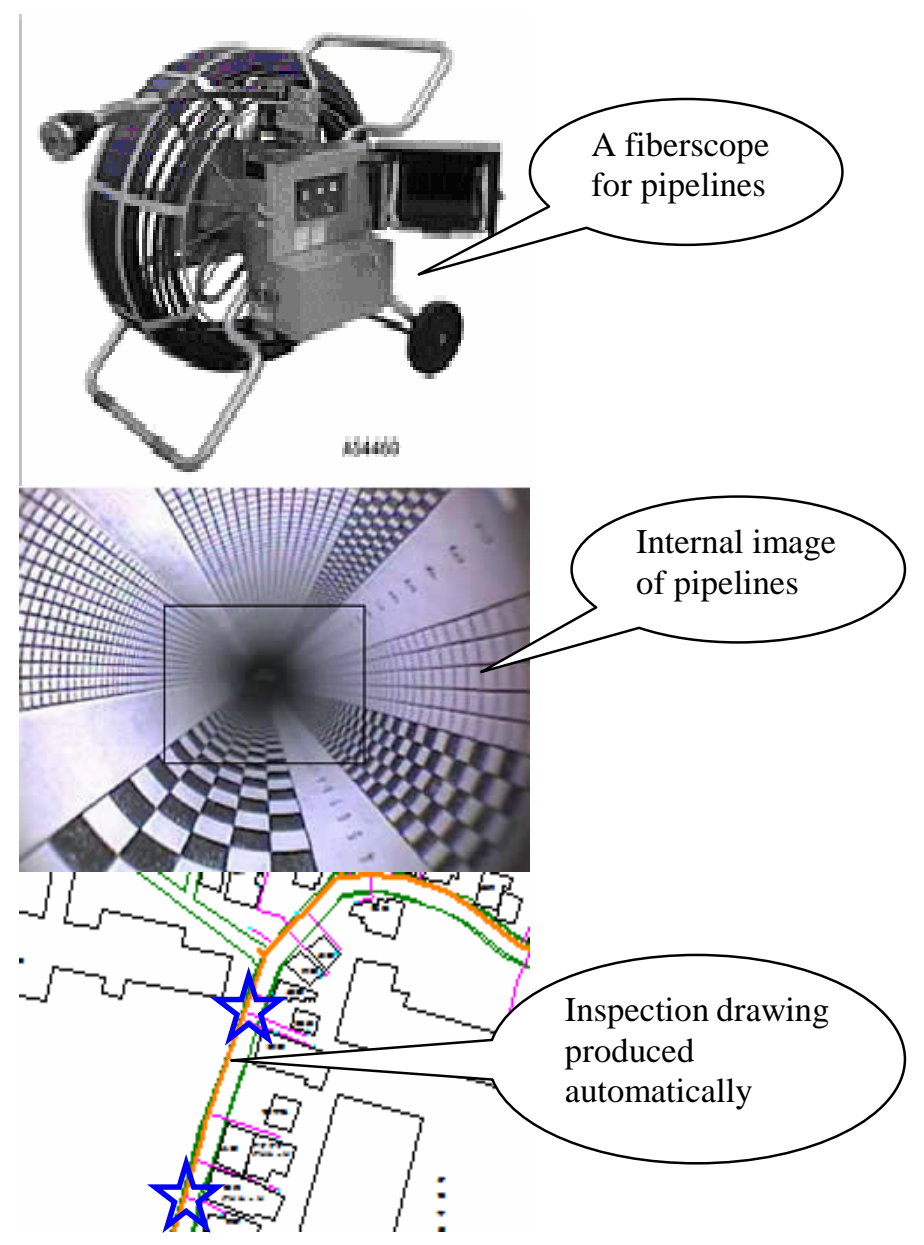

Fig.12 a fiberscope system \& GIS integration

\section{CONCLUSION}

TUMSY has been implemented for about 30 years in Tokyo Gas and other utilities. By both simple architecture and continuous and updating, it has been useful and necessary to users.

The history tells us that Tokyo Gas GIS is essential to the company activities, and must be always implemented on the highest level of technology and business expertise.

\section{REFERENCES}

[1] http://www.tumsy.com/

[2] http://www.roadic.or.jp/

[3] http://www.geoap.jp/ 